\title{
ON REPRESENTABLE PAIRS
}

\section{Tomáš Kepka, Petr Němec and Markku Niemenmaa}

\section{Introduction}

This paper is organised in five parts. We first consider some properties of semigroups in Chapter 1 and prove structural results which might be interesting as such. In Chapter 2 we define our central notion, namely, that of a representable pair $(S, f)$ where $S$ is a semigroup and $f$ is a mapping from $S$ into the class of non-zero cardinal numbers. We also give here some necessary conditions for a pair $(S, f)$ to be representable by a groupoid.

Chapter 3 contains a representation criterion. By using this criterion we are able to prove in Chapter 4 several sufficient conditions for a pair $(S, f)$ to be representable by a groupoid. Finally, Chapter 5 contains a special treatment of a semigroup of order five.

We assume that the reader is familiar with the rudiments of the theory of abstract algebraic systems. The background can be obtained e.g. from [1], [2] and $[3]$.

\section{Preliminaries}

Let $S$ be a semigroup. We denote $S^{2}=S S=\{a b: a, b \in S\}$ and $S^{n}=$ $S S^{n-1}$ for every positive integer $n \geq 3$. We also need the following sets:

$$
\begin{aligned}
\operatorname{Id}(S) & =\left\{a \in S: a=a^{2}\right\}, \\
L(S) & =\{a \in S: a \in S a\}, \\
R(S) & =\{a \in S: a \in a S\}, \\
\operatorname{Li}(S) & =\{a \in S: a \in \operatorname{Id}(S) a\}, \\
\operatorname{Ri}(S) & =\{a \in S: a \in a \operatorname{Id}(S)\}, \\
K(S) & =\bigcap_{n=1}^{\infty} S^{n} .
\end{aligned}
$$

We shall now formulate some easy observations.

Lemma 1.1. (i) The set $L(S)$ (or $R(S)$ ) is either empty or a right (or left) ideal of $S$.

(ii) The set $\operatorname{Li}(S)$ (or $\operatorname{Ri}(S)$ ) is either empty or a right (or left) ideal of $S$. 
(iii) The set $K(S)$ is either empty or an ideal of $S$.

(iv) $\operatorname{Id}(S) \subseteq \operatorname{Li}(S) \subseteq L(S) \subseteq K(S)$ and $\operatorname{Id}(S) \subseteq \operatorname{Ri}(S) \subseteq R(S) \subseteq K(S)$.

If there exists an integer $n \geq 0$ such that $S^{n-1} \neq S^{n}=K(S)$, we say that the number $n=n c(S)$ is the class number of $S$ (now $S^{0}$ means a one-element semigroup and $S^{-1}=\emptyset$ ).

Lemma 1.2. If $S$ is finite, then $\operatorname{Id}(S)$ is non-empty, $L(S)=\operatorname{Li}(S)$ and $R(S)=\operatorname{Ri}(S)$. Furthermore, $K(S)^{2}=K(S)$.

Lemma 1.3. Let $S$ be finite and $S=S^{2}$ (i.e., $n c(S) \leq 1$ ). Then $S=$ $R(S) L(S)$. In particular, $S=L(S)$, provided that $S$ is commutative.

Proof. Put $I=R(S) L(S)$ and define a relation $r$ on $S$ by $(a, b) \in r$ if and only if $a \in b S$. Now $I$ is an ideal of $S, r$ is transitive and $a \in R(S)$ if and only if $(a, a) \in r$. Then assume that $a_{1} \in S-I$. There are elements $a_{2}, b_{1} \in S$ such that $a_{1}=a_{2} b_{1}$, also $a_{3}, b_{2} \in S$ such that $a_{2}=a_{3} b_{2}$ etc. Now $\left(a_{1}, a_{2}\right) \in r,\left(a_{2}, a_{3}\right) \in r$ etc., so that $\left(a_{i}, a_{j}\right) \in r$ whenever $1 \leq i<j$. Since $I$ is an ideal and $a_{1} \notin I$, we conclude that $I$ contains none of the elements $a_{2}, a_{3}, \ldots$ As $S$ is finite, it follows that there are positive integers $i<j$ such that $a_{i}=a_{j}$. Thus $\left(a_{i}, a_{i}\right) \in r$, $a_{i} \in R(S)$, and since $R(S) \subseteq I$ by $1.2(\mathrm{i})$, we get $a_{i} \in I$, a contradiction. The proof is complete.

Lemma 1.4. Suppose that $S$ contains at most four elements and $S=S^{2}$. Then $S=L(S) \cup R(S)$.

Proof. Suppose that $a \in S$ and $a \notin L(S) \cup R(S)$. By 1.3, $a=b c$, where $b \in R(S)$ and $c \in L(S)$. Clearly, $b \notin L(S)$ and $c \notin R(S)$. Now the elements $a, a^{2}, b$ and $c$ are pair-wise different, hence $\operatorname{card}(S)=4$. If $b a=b$, then $a=$ $b c=b a c=b^{2} c^{2}=b^{3} c^{3}=\ldots ;$ now $b^{n} \in \operatorname{Id}(S)$ for some $n \geq 1$, the equation $a=b^{n} c^{n}$ implying $a \in L(S)$, a contradiction. Similarly, if $b a^{2}=b$, we get a contradiction. Consequently, $b a \neq b$ and $b a^{2} \neq b$. The inequalities $b b \neq b$ and $b c \neq b$ are obvious; thus we have proved that $b \notin b S$. Hence $b \notin R(S)$ and we again have a contradiction. We conclude $S=L(S) \cup R(S)$.

Example 1.5. Consider the following five-element semigroup $T$ :

\begin{tabular}{c|ccccc} 
& 0 & $a$ & $b$ & $c$ & $d$ \\
\hline 0 & 0 & 0 & 0 & 0 & 0 \\
$a$ & 0 & 0 & 0 & 0 & 0 \\
$b$ & 0 & 0 & 0 & $a$ & $b$ \\
$c$ & 0 & 0 & 0 & 0 & 0 \\
$d$ & 0 & 0 & 0 & $c$ & $d$
\end{tabular}

Now $T=T^{2}$ and $a \notin L(T) \cup R(T)$. 
Lemma 1.6. Let $S$ be a five-element semigroup such that $S=S^{2}$ and $S \neq L(S) \cup R(S)$; then $S$ is isomorphic to the semigroup $T$ constructed in 1.5.

Proof. Let $a \in S-(L(S) \cup R(S))$. Now $a=b c$, where $b \in R(S)$ and $c \in L(S)$. Furthermore, $b \notin L(S)$ and $c \notin R(S)$. The elements $a, a^{2}, b$ and $c$ are pair-wise different and, as in the proof of Lemma 1.4, one can show that $b \notin\left\{b a, b a^{2}, b b, b c\right\}$. Since $b \in R(S), b=b d$, where $d \in S$ and thus $S=\left\{a, a^{2}, b, c, d\right\}$. By using a similar type of argument, we get $c=d c$.

Now we know that $b=b d$ and $c=d c$ and we try to compute the rest of the multiplication table for $S$. It is easy to see that $a b \neq a, a b \neq b, a b \neq c$ and $a b \neq d$; hence $a b=a^{2}$. We also have $b a=a^{2}, a c=a^{2}$ and $c a=a^{2}$. Further, it is easy to see that $b^{2}=a^{2}$ and $c^{2}=a^{2}$. Clearly, $a d \neq a$ and $a d \neq b$. If $a d=c$, then $a=b c=b a d=b^{2} a d^{2}=\cdots$, a contradiction. If $a d=d$, then $b=b d=b a d$ and $a=b c=b a d c=b^{2} a(d c)^{2}=\cdots$, again a contradiction. Consequently, $a d=a^{2}$ and, similarly, $d a=a^{2}$. Since $a \notin R(S) \cup L(S), b \notin L(S)$ and $c \notin R(S)$, we must have $c b=a^{2}, c d=a^{2}$ and $d b=a^{2}$. Clearly, $a^{3} \neq a, a^{3} \neq b$ and $a^{3} \neq c$. If $a^{3}=d$, then $b=b d=b a^{3}$ and $a=b c=b a^{3} c$, which is not possible. Thus $a^{3}=a^{2}$, and it follows that $a^{4}=a^{2}, b a^{2}=a^{2} b=c a^{2}=a^{2} c=d a^{2}=a^{2} d=a^{2}$. Finally, we have to see that $d^{2}=d$. If we denote $a^{2}=0$, we have the same multiplication table as in the example. The proof is complete.

\section{Groupoids and semigroups}

By a groupoid we mean a non-empty set together with a binary operation denoted multiplicatively.

Let $\zeta$ be a groupoid. Denote by $r(\zeta)$ the intersection of all congruences $r$ such that the corresponding factor groupoid is a semigroup. Now $r(\zeta)$ is a congruence on $\zeta, \zeta / r(\zeta)$ is a semigroup and $r(\zeta)$ is the least congruence with this property. Clearly, $r(\zeta)$ is the congruence on $\zeta$ generated by the ordered pairs $(a(b c),(a b) c)$, where $a, b, c \in \zeta$.

Throughout the paper, let $C$ denote the class of non-zero cardinal numbers. Consider a semigroup $S$ and a mapping $f: S \rightarrow C$. We say that the pair $(S, f)$ is representable by a groupoid if there exist a groupoid $\zeta$ and a homomorphism $g$ from $\zeta$ onto $S$ such that $\operatorname{ker}(g)=r(\zeta)$ and $\operatorname{card}\left(g^{-1}(a)\right)=f(a)$ for every $a \in S$.

We immediately have

Lemma 2.1. Let $S$ be a semigroup and $f: S \rightarrow C$ a mapping such that the pair $(S, f)$ is representable by a groupoid. Then $f(a)=1$ for every $a \in S-S^{3}$.

Next we establish

Lemma 2.2. Let $S$ be a semigroup and $f: S \rightarrow C$ a mapping such that the pair $(S, f)$ is representable by a groupoid. Let $a \in S^{2}$ and

$$
A=\{(b, c) \mid b, c \in S \text { and } a=b c\} .
$$

Then $f(a) \leq \sum_{(b, c) \in A} f(b) f(c)$. 
Proof. Suppose that

$$
f(a)>\sum_{(b, c) \in A} f(b) f(c)
$$

Now there exist a groupoid $\zeta$ and a surjective homomorphism $g: \zeta \rightarrow S$ such that $\operatorname{ker}(g)=r(\zeta)$ and $\operatorname{card}\left(g^{-1}(u)\right)=f(u)$ for every $u \in S$. Put $H=g^{-1}(a)$ and

$$
L=\left\{x y \mid x \in g^{-1}(b), y \in g^{-1}(c),(b, c) \in A\right\} .
$$

Then $L \subseteq H$ and $L \neq H$. Now we define a relation $d$ on $\zeta$ as follows:

$$
d=(r(\zeta)-(H \times H)) \cup(L \times L) \cup((H-L) \times(H-L)) .
$$

Clearly, $d$ is an equivalence relation, $d \subseteq r(\zeta)$ and $d \neq r(\zeta)$. In fact, it is not difficult to see that $d$ is a congruence on $\zeta$ and $\zeta / d$ is a semigroup. However, this is a contradiction. The required result follows.

\section{A representation criterion}

Let $S$ be a semigroup and $f: S \rightarrow C$ a mapping. For each $a \in S$, define a mapping $f_{a}: S \rightarrow C$ by $f_{a}(a)=f(a)$ and $f_{a}(b)=1$ for every $b \neq a, b \in S$.

Theorem 3.1. Suppose that the pair $\left(S, f_{a}\right)$ is representable by a groupoid for any $a \in S$. Then the pair $(S, f)$ is also representable by a groupoid.

Proof. Now there exist pair-wise disjoint groupoids $\zeta_{a}$ (their operations are denoted by $\star$ ) and surjective homomorphisms

$$
g_{a}: \zeta_{a} \rightarrow S
$$

such that

$$
\begin{aligned}
& \operatorname{ker}\left(g_{a}\right)=r\left(\zeta_{a}\right) \\
& \operatorname{card}\left(g_{a}^{-1}(a)\right)=f(a) \text { and } \\
& \operatorname{card}\left(g_{a}^{-1}(b)\right)=1 \quad \text { for every } b \in S, b \neq a .
\end{aligned}
$$

Now denote $H_{a}=g_{a}^{-1}(a)$ and $\zeta=\bigcup_{a \in S} H_{a}$. We shall define a binary operation on $\zeta$ as follows:

(1) If $x, y \in H_{a}$ and $a=a a$, we put $x y=x \star y \in H_{a}$.

(2) If $x \in H_{a}, y \in H_{b}$ and $a b=c$ where $a \neq c \neq b$, then $x y=g_{c}^{-1}(a) \star$ $g_{c}^{-1}(b) \in H_{c}$.

(3) If $x \in H_{a}$ and $y \in H_{b}(a \neq b)$ and $a b=a$, then $x y=x \star g_{a}^{-1}(b) \in H_{a}$.

(4) If $x \in H_{a}$ and $y \in H_{b}(a \neq b)$ and $a b=b$, we put $x y=g_{b}^{-1}(a) \star y \in H_{b}$. 
Now we define a mapping $g$ from $\zeta$ onto $S$ by

$$
g\left(H_{a}\right)=a \quad \text { for each } a \in S .
$$

It is obvious that $g$ is a homomorphism from $\zeta$ to $S$.

We still have to show that $r(\zeta)=\operatorname{ker}(g)$. Clearly, $r(\zeta) \subseteq \operatorname{ker}(g)$. We shall now construct two equivalence relations for our proof. Let $a \in S$ and denote

$$
d=\left(\operatorname{ker}(g)-\left(H_{a} \times H_{a}\right)\right) \cup\left(r(\zeta) \cap\left(H_{a} \times H_{a}\right)\right)
$$

and

$$
s=\left\{(x, x) \mid x \in \zeta_{a}\right\} \cup\left(r(\zeta) \cap\left(H_{a} \times H_{a}\right)\right) .
$$

We are going to prove that $d$ is a congruence on $\zeta$ and $s$ is a congruence on $\zeta_{a}$.

Let $x, y, z \in \zeta$ and $(x, y) \in d$. We have to distinguish between the following cases:

(1) $x, y \in H_{b}$ for some $b \in S, b \neq a$. Then $(z x, z y) \in \operatorname{ker}(g)$ and $(z x, z y) \in d$ unless $z x \in H_{a}$. If $z x \in H_{a}$, then $z y \in H_{a}$, too. Then there exists $c \in S$ such that $z \in H_{c}$ and $a=c b$. If $a \neq c$, then

$$
z x=g_{a}^{-1}(c) \star g_{a}^{-1}(b)=z y,
$$

hence $(z x, z y) \in d$. If $a=c$, then

$$
z x=z \star g_{a}^{-1}(b)=z y
$$

and again $(z x, z y) \in d$.

(2) $x, y \in H_{a}$ and $(x, y) \in r(\zeta)$. If $z x \notin H_{a}, z y \notin H_{a}$, then $(z x, z y) \in \operatorname{ker}(g)$, hence $(z x, z y) \in d$. Now consider the case where $z x \in H_{a}$ and $z y \in H_{a}$. Then, clearly, $(z x, z y) \in r(\zeta) \cap\left(H_{a} \times H_{a}\right)$, hence $(z x, z y) \in d$.

Now we have proved that $(z x, z y) \in d$ (in a similar way we could prove that $(x z, y z) \in d)$. Thus $d$ is a congruence on $\zeta$.

After this we shall have a look at the relation $s$. Let $x, y, z \in \zeta_{a}$ and $(x, y) \in s$. Now we have to consider the following three cases:

(1) $x \notin H_{a}$. Then $y \notin H_{a}, x=y$ and $(z \star x, z \star y) \in s$.

(2) $x \in H_{a}$ and $z \star x \notin H_{a}$. Now $y \in H_{a},(x, y) \in \operatorname{ker}\left(g_{a}\right),(z \star x, z \star y) \in \operatorname{ker}\left(g_{a}\right)$ and thus $z \star x=z \star y$ implying that $(z \star x, z \star y) \in s$.

(3) $x \in H_{a}$ and $z \star x \in H_{a}$. Then $y \in H_{a}, z \star y \in H_{a}$, and naturally $(x, y) \in r(\zeta)$. Now put $b=g_{a}(z)$ so that $a=b a$. If $b \neq a$ (this means that $\left.z \notin H_{a}\right)$, then $(u x, u y) \in r(\zeta)$ for any $u \in H_{b}$, and, moreover, $u x=z \star x$ and $u y=z \star y$, hence $(z \star x, z \star y) \in s$. 
If $b=a$ (then $z \in H_{a}$ ), we have $(z x, z y) \in r(\zeta)$ and now $z x=z \star x$ and $z y=z \star y$. Once again $(z \star x, z \star y) \in s$, and we thus have shown that $s$ is a congruence on $\zeta_{a}$.

Now it is clear that $r(\zeta) \subseteq d \subseteq \operatorname{ker}(g)$. There exist the projective natural homomorphisms

and a homomorphism

$$
\begin{aligned}
& p: \zeta \rightarrow \zeta / r(\zeta), \\
& q: \zeta / r(\zeta) \rightarrow \zeta / d
\end{aligned}
$$

$$
k: \zeta / d \rightarrow S
$$

such that $g=k q p$.

Since $s \subseteq \operatorname{ker}\left(g_{a}\right)$ we also have the projective natural homomorphism

$$
f: \zeta_{a} \rightarrow \zeta_{a} / s
$$

and a homomorphism

$$
v: \zeta_{a} / s \rightarrow S
$$

such that $g_{a}=v f$.

Finally, define a mapping $h: \zeta \rightarrow \zeta_{a}$ by

$$
h(x)= \begin{cases}x & \text { if } x \in H_{a} \\ g_{a}^{-1}(b) & \text { if } x \in H_{b}, b \neq a .\end{cases}
$$

The mapping $h$ thus defined is a homomorphism from $\zeta$ onto $\zeta_{a}$, and we now have the following commutative diagram:

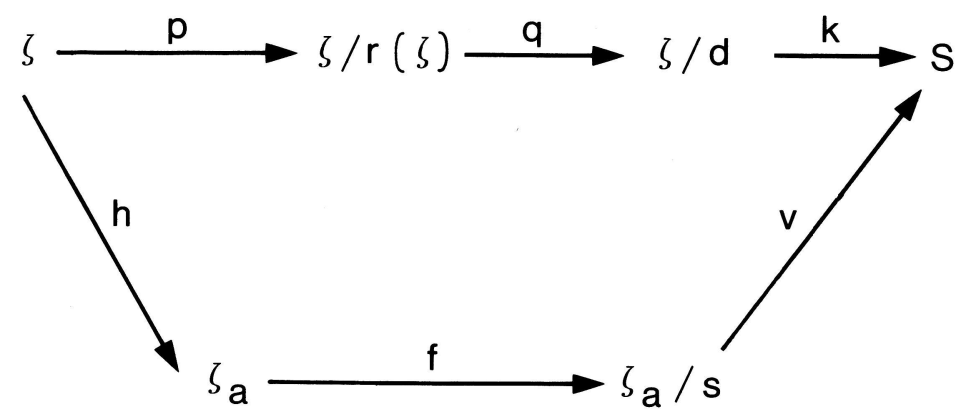

It is easily verified that $\operatorname{ker}(f h)=d=\operatorname{ker}(q p)$, from which it follows that the groupoids $\zeta / d$ and $\zeta_{a} / s$ are isomorphic. Since $\zeta / d$ is a homomorphic image of $\zeta / r(\zeta)$, it is a semigroup and it follows that $\zeta_{a} / s$ is a semigroup, too.

We first conclude that $s=\operatorname{ker}\left(g_{a}\right)$ and then $r(\zeta) \cap\left(H_{a} \times H_{a}\right)=H_{a} \times H_{a}$. This implies that $r(\zeta) \supseteq H_{a} \times H_{a}$, hence $r(\zeta)=\operatorname{ker}(g)$. The proof is complete. 


\section{Some representable pairs}

We shall now establish some representable pairs by using the results of the preceding chapters. However, we first prove two preliminary lemmas.

Lemma 4.1. Let $M$ be a non-empty set. Then there exists a mapping $t$ from $M$ onto $M$ such that for all $x, y \in M$ there exist positive integers $m, n$ such that $t^{m}(x)=t^{n}(y)$.

Proof. (1) Let $k$ be a positive integer. Now the permutation $t(1)=2$, $t(2)=3, \ldots, t(k-1)=k, t(k)=1$ on the set $\{1,2, \ldots, k\}$ has the desired property.

(2) If we consider $\mathbf{N}$, we define $t(k)=k-1$ for every $k \geq 2$ and $t(1)=1$.

(3) Let $a$ be an infinite cardinal number, $A$ be a set with $\operatorname{card}(A)=a$ and $B$ be the set of all mappings $f: A \rightarrow \mathbf{N}$ with $f(x) \neq 1$ only for a finite number of elements $x$ from $A$. Define a mapping $t$ from $B$ onto $B$ by $t(f)(x)=1$ if $f(x)=1$ and $t(f)(x)=f(x)-1$ if $f(x) \geq 2$. Again, $t$ has the desired property.

Lemma 4.2. Let $S$ be a semigroup, $a \in L(S)$ (or $a \in R(S)$ ) and let $f: S \rightarrow C$ be a mapping such that $f(b)=1$ for every $b \in S-\{a\}$. Then the pair $(S, f)$ is representable by a groupoid.

Proof. We shall now exceptionally denote the operation of $S$ by $(\star)$. Denote further $R=S-\{a\}$ and let $M$ be a set with $\operatorname{card}(M)=f(a)$ and $S \cap M=\emptyset$. Finally, let $\zeta=R \cup M$.

Since $a \in L(S)$, there exists $e \in S$ such that $e \star a=a$. We first assume that $a \notin \operatorname{Id}(S)$ and define a multiplication on $\zeta$ as follows:

(1) $e x=(e \star e)=t(x)$ for every $x \in M$, where $t$ is a mapping from $M$ onto $M$ as given in Lemma 4.1,

(2) $b c=b \star c$ for all $b, c \in R$ with $b \star c \neq a$,

(3) $b c=$ any element of $M$ if $b, c \in R$ and $b \star c=a$,

(4) $b x=b \star a$ if $b \in R, x \in M$ and $b \star a \neq a$,

(5) $b x=$ any element of $M$ if $b \in R, b \neq e, b \neq e \star e, x \in M$ and $b \star a=a$,

(6) $x b=a \star b$ if $b \in R, x \in M$ and $a \star b \neq a$,

(7) $x b=$ any element of $M$ if $b \in R, x \in M$ and $a \star b=a$,

(8) $x y=a \star a$ for all $x, y \in M$.

After this we define a mapping from $\zeta$ onto $S$ by $g(x)=x$ if $x \in R$ and $g(x)=a$ if $x \in M$. Clearly, $g$ is a homomorphism. It remains to show that $\operatorname{ker}(g)=r(\zeta)$. Once again, it is obvious that $r(\zeta) \subseteq \operatorname{ker}(g)$. Put $s=r(\zeta) \cap(M \times M)$. If $(x, y) \in s$, then $(t(x), t(y))=(e x, e y) \in s$, which means that $s$ is a congruence on the algebra $(M, t)$ with one unary operation. If $x \in M$, then, by the definition of $r(\zeta),(e(e x),(e e) x) \in r(\zeta)$. Now $e(e x)=t^{2}(x)$ and $(e e) x=t(x)$, hence $\left(t^{2}(x), t(x)\right) \in s$. In fact, if $n$ is a positive integer, then $\left(t^{n}(x), t(x)\right) \in s$. Let $(u, v) \in M \times M$. Now there exist elements $a, b \in M$ such that $u=t(a)$ and $v=t(b)$. By Lemma 4.1, there also exist positive integers $m, n$ such that 
$t^{m}(a)=t^{n}(b)$. On the other hand, $\left(t^{m}(a), t(a)\right) \in s$ and $\left(t^{n}(b), t(b)\right) \in s$, hence $(t(a), t(b))=(u, v) \in s$. It follows that $s=M \times M$. Then certainly $r(\zeta) \supseteq M \times M$ and $\operatorname{ker}(g)=r(\zeta)$.

Then assume that $a \in \operatorname{Id}(S)$. Choose an element $w \in M$ and define a multiplication on $\zeta$ as follows:

(1) $x y=w$ for all $x, y \in M, y \neq w$,

(2) $x w=x$ for every $x \in M$,

(3) $b c=b \star c$ for all $b, c \in R$ with $b \star c \neq a$,

(4) $b c=w$ if $b, c \in R$ and $b \star c=a$,

(5) $b x=b \star a$ if $b \in R, x \in M$ and $b \star a \neq a$,

(6) $b x=w$ if $b \in R, x \in M$ and $b \star a=a$,

(7) $x b=a \star b$ if $b \in R, x \in M$ and $a \star b \neq a$,

(8) $x b=w$ if $b \in R, x \in M$ and $a \star b=a$.

Then we define a mapping $g$ from $\zeta$ onto $S$ as in the first part of the proof. Naturally, $g$ is a homomorphism. Suppose that $(x, y) \in M \times M$. By the definition of $r(\zeta),(x(w x),(x w) x) \in r(\zeta)$, i.e., $(x, w) \in r(\zeta)$. Similarly, $(y, w) \in r(\zeta)$, hence $(x, y) \in r(\zeta)$. Now again $r(\zeta) \supseteq M \times M$ and $\operatorname{ker}(g)=r(\zeta)$. This completes the proof.

We are now able to give our first theorem about representable pairs.

Theorem 4.3. Let $S$ be a semigroup such that $S=L(S) \cup R(S)$. Then the pair $(S, f)$ is representable by a groupoid for any mapping $f: S \rightarrow C$.

Proof. Just combine Theorem 3.1 with Lemma 4.2.

By using Lemmas 1.3 and 1.4 we immediately have

Corollary 4.4. The pair $(S, f)$ is representable by a groupoid for any mapping $f: S \rightarrow C$ if

(i) $S$ is finite, commutative and $n c(S) \leq 1$, or

(ii) $S$ contains at most four elements and $n c(S) \leq 1$.

The rest of this chapter is devoted to the investigation of the situation where $f(S)=\{1,2\}$. We first prove

Theorem 4.5. Let $S$ be a semigroup, $a \in S$ and let $f: S \rightarrow C$ be a mapping such that $f(a)=2$ and $f(b)=1$ for every $b \in S, b \neq a$. Then the pair $(S, f)$ is representable by a groupoid if and only if at least one of the following two conditions is satisfied:

(i) $a \in L(S) \cup R(S)$,

(ii) there exist $x, y, z \in S$ such that $a=x y z$ and either $x \neq x y$ or $z \neq y z$.

Proof. If (i) holds, the result follows from Lemma 4.2.

Then assume that (ii) holds: i.e., $a=x y z$ with $x \neq x y$. Now take an element $e \notin S$ and put $\zeta=S \cup\{e\}$. An operation $(\star)$ on $\zeta$ is defined in the following manner: 
(1) $u \star v=u v$ for all $u, v \in S$ with $u v \neq a$,

(2) $u \star v=a$ for all $u, v \in S$ with $u v=a$, and either $u \neq x$ or $v \neq y z$,

(3) $x \star y z=e$,

(4) $e \star u=a \star u$ and $u \star e=u \star a$ for every $u \in S$,

(5) $e \star e=a \star a$.

Define a mapping $g$ from $\zeta$ onto $S$ by $g(e)=a$ and $g(x)=x$ for every $x \in \zeta-\{e\}$. Clearly, $g$ is a homomorphism and $\operatorname{ker}(g)=r(\zeta)$.

The case where (ii) holds, $a=x y z$ and $z \neq y z$ proceeds in a similar way.

Now we prove the converse statement. Suppose that the pair $(S, f)$ is representable by a groupoid. This means that we have a groupoid $\zeta$ and a homomorphism $g: \zeta \rightarrow S$ such that $\operatorname{ker}(g)=r(\zeta), \operatorname{card}\left(g^{-1}(a)\right)=2$ and $\operatorname{card}\left(g^{-1}(b)\right)=1$ for every $b \in S, b \neq a$. Assume that neither (i) nor (ii) is true. Let $u, v, w$ be elements of $\zeta$ and $x=u v, y=v w$. If $g(u y) \neq a$, then also $g(x w) \neq a$, hence $u y=x w$. If $g(u y)=a$, then $g(x w)=a$ and we have $a=g(u) g(v) g(w)$. Since (ii) does not hold, $g(u)=g(u) g(v)=g(x)$ and $g(w)=g(v) g(w)=g(y)$. Since $a \notin L(S) \cup R(S), g(u) \neq a \neq g(w)$, yielding $u=x$ and $w=y$. But then $u(v w)=u y=u w=x w=(u v) w$, and we have shown that $\zeta$ is a semigroup, a contradiction. Thus either (i) or (ii) is true as required.

By combining Theorems 3.1 and 4.5 we get

Corollary 4.6. Let $S$ be a semigroup such that for every $a \in S-(L(S) \cup$ $R(S))$ there exist elements $x, y, z \in S$ with $a=x y z$ and $(x, y z) \neq(x y, z)$. Then the pair $(S, f)$ is representable by a groupoid for any mapping $f: S \rightarrow\{1,2\}$.

It is also easy to see that the following result (which is in fact partially converse to Lemma 2.1) is true.

Corollary 4.7. Let $S$ be a commutative semigroup and $f: S \rightarrow\{1,2\}$ a mapping. Then the pair $(S, f)$ is representable by a groupoid if $f(a)=1$ for every $a \in S-S^{3}$.

\section{An example}

In Lemma 2.2 we proved that if the pair $(S, f)$ is representable by a groupoid and $a \in S^{2}$, then

$$
f(a) \leq \sum f(b) f(c)
$$

where we go through all elements $b, c \in S$ such that $b c=a$. In what follows we consider the semigroup $T=\{0, a, b, c, d\}$ from Example 1.5 and show that $(T, f)$ is representable by a groupoid if $f(a) \leq f(b) f(c)$.

Let us assume that $f(a) \leq f(b) f(c)$. Then take five pair-wise disjoint sets $P, A, B, C$ and $D$ such that $\operatorname{card}(P)=f(0), \operatorname{card}(A)=f(a), \operatorname{card}(B)=f(b)$, $\operatorname{card}(C)=f(c)$ and $\operatorname{card}(D)=f(d)$. Put $\zeta=P \cup A \cup B \cup C \cup D$ and let $p: B \rightarrow B$ and $q$ : $C \rightarrow C$ be mappings described in Lemma 4.1. From our assumption it 
follows that there exists a mapping $h$ from $B \times C$ onto $A$. Now choose elements $z \in P$ and $w \in D$ and define a multiplication on $\zeta$ as follows:

(1) $x y=y x=z$ for every $x \in P$ and $y \in A \cup B \cup C \cup D$,

(2) $x y=z$ for all $x, y \in A \cup B$,

(3) $x y=y x=z$ for every $x \in A$ and $y \in C \cup D$,

(4) $x y=z$ for every $x \in C$ and $y \in B$,

(5) $x y=z$ for all $x, y \in C$,

(6) $x y=z$ for every $x \in C$ and $y \in D$,

(7) $x y=z$ for every $x \in D$ and $y \in B$,

(8) $x y=z$ for all $x, y \in P \quad(y \neq z)$,

(9) $x z=x$ for every $x \in P$,

(10) $x y=w$ for all $x, y \in D \quad(y \neq w)$,

(11) $x w=x$ for every $x \in D$,

(12) $x y=p(x)$ for every $x \in B$ and $y \in D$,

(13) $x y=q(y)$ for every $x \in D$ and $y \in C$,

(14) $x y=h(x, y)$ for every $x \in B$ and $y \in C$.

Then define a mapping $g$ from $\zeta$ onto $T$ by $g(P)=0, g(A)=a, g(B)=b$, $g(C)=c$ and $g(D)=d$. It is easy to check that $g$ is a homomorphism. We now have to show that $r(\zeta)=\operatorname{ker}(g)$.

First, $(x(x x),(x x) x) \in r(\zeta)$ for any $x \in P$. Since $x(x x)=x z=x$ and $(x x) x=z x=z$, it follows that $(x, z) \in r(\zeta)$, hence $P \times P \subseteq r(\zeta)$. Similarly, one can prove that $D \times D \subseteq r(\zeta)$. The inclusions $B \times B \subseteq r(\zeta)$ and $C \times C \subseteq r(\zeta)$ can be proved as in Lemma 4.2 (now the mappings $p$ and $q$ have the role of $t$ ). Finally, if $(x, y) \in B \times B$ and $(u, v) \in C \times C$, then also $(x, y) \in r(\zeta)$ and $(u, v) \in r(\zeta)$, hence $(x u, y v) \in r(\zeta)$. By definition $(h(x, u), h(y, v)) \in r(\zeta)$, and thus $A \times A \subseteq r(\zeta)$. We conclude that $\operatorname{ker}(g)=r(\zeta)$, and the proof is complete.

\section{References}

[1] Howie, J.M.: An introduction to semigroup theory. - Academic Press, London-New YorkSan Francisco, 1976.

[2] Koskas, M.: Groupoides, demi-groupes et hypergroupes. - J. Math. Pures et Appl. 49, 1970, 155-192.

[3] Sigler, L.E.: Algebra. - Springer-Verlag, New York-Heidelberg-Berlin, 1976.

Charles University

Department of Mathematics 18600 Prague 8

Czechoslovakia

Received 1 April 1987
Charles University

Department of Mathematics

18600 Prague 8

Czechoslovakia
University of Oulu

Department of Mathematics

SF-90570 Oulu

Finland 